\title{
Is all risk bad? Young adult cigarette smokers fail to take adaptive risk in a laboratory decision-making test
}

\author{
Andy C. Dean - Catherine A. Sugar • \\ Gerhard Hellemann • Edythe D. London
}

Received: 3 September 2010 /Accepted: 12 January 2011 / Published online: 5 February 2011

(C) The Author(s) 2011. This article is published with open access at Springerlink.com

\begin{abstract}
Rationale Cigarette smoking has been linked to real-world risky behavior, but this association has been based largely on retrospective self-reports. Limitations of self-report data can be avoided by using laboratory, performance-based measures, such as the Balloon Analogue Risk Task (BART; Lejuez et al., J Exp Psychol Appl 8:75-84, 2002). Initial studies have suggested that smokers display greater risktaking on this task than nonsmokers, but these studies did not account for drug abuse and psychiatric comorbidities, which are commonplace among smokers.

Objectives We sought to examine the performance of smokers and nonsmokers on the BART after excluding drug abuse and psychiatric comorbidities.

Methods We conducted a study of late adolescent/young adult (age 18 to 21$)$ smokers $(n=26)$ and nonsmokers $(n=38)$ performing the BART and excluded individuals with positive
\end{abstract}

\footnotetext{
A. C. Dean · E. D. London

Department of Psychiatry and Biobehavioral Sciences and Semel Institute, David Geffen School of Medicine,

University of California,

Los Angeles, CA 90095, USA

C. A. Sugar • G. Hellemann

Department of Biostatistics, UCLA School of Public Health, P.O. Box 591772, Los Angeles, CA 90095, USA

E. D. London

Department of Molecular and Medical Pharmacology, and the Brain Research Institute,

David Geffen School of Medicine, University of California, Los Angeles, CA 90095, USA

E. D. London $(\bowtie)$

UCLA Semel Institute for Neuroscience and Human Behavior, 760 Westwood Plaza,

Los Angeles, CA 90095-1759, USA

e-mail: elondon@mednet.ucla.edu
}

drug or alcohol toxicology screens, substance abuse or dependence diagnoses, and/or current psychiatric conditions. Results Contrary to previous findings, smokers did not display greater risk-taking on the BART than nonsmokers. In fact, when performance was examined trial-by-trial, the nonsmokers displayed progressively greater pumping relative to smokers over time $(p<.001)$, earning them a nonsignificantly greater amount of money than the smokers. Controlling for smoking status, additional analyses revealed that pumping on the BART was positively associated with years of education, nonverbal IQ, and employment.

Conclusions The results suggest that in young adults, smoking may be associated with a failure to take risks in situations where risk-taking is adaptive.

Keywords Cigarette smoking - Adolescent - Young adult . Risk $\cdot$ Impulsivity $\cdot$ Adaptive

Cigarette smoking is a risky behavior. Despite potential short-term benefits such as improved mood and acceptance from peers, the long-term consequences of smoking include a variety of serious medical problems and other morbidities (CDC 2002, 2005). Cigarette smoking represents one of the six categories of priority health-risk behaviors regularly monitored in the Youth Risk Behavior Surveillance System (YRBSS) through the Centers for Disease Control and Prevention (CDC), with approximately $20 \%$ of high school students endorsing cigarette use within the last 30 days during the most recent surveillance period (Youth Risk Behavior Surveillance, USA, 2009 (Eaton et al. 2010)).

Several lines of research have suggested that in addition to the inherent risks presented by smoking behavior, individuals who smoke may be more likely than nonsmokers to engage in other forms of risky behavior. For example, compared to nonsmokers, smokers are more likely to use alcohol and illicit substances (Hanna and Grant 1999), be employed in risky occupations (Hersch and 
Viscusi 1998), have traffic accidents (DiFranza et al. 1986), and not wear a seatbelt while driving (Ryb et al. 2007). Such risky behavior may be particularly salient during adolescence and young adulthood. For instance, smokers in high school and college are more likely to engage in risky sexual behaviors than nonsmokers (Schneider and Morris 1991; Valois 1999), and female teenagers with a history of smoking are more likely than never-smoking teens to become pregnant (Seamark and Gray 1998). Moreover, between the ages of 16 to 29 , smokers are twice as likely to present to a hospital emergency room (ER) for treatment than are their non-smoking peers (Indig et al. 2007).

Although the aforementioned studies suggest that risktaking is associated with smoking behavior, most of the cited evidence was obtained through self-report and/or through the endorsement of behaviors that are presumed to reflect risk-taking tendencies (e.g., drug use and ER visits). Because self-report biases may be problematic when assessing risky behaviors that are socially discouraged (e.g., Fitzgerald and Mulford 1987; Kypri 2007), Lejuez and colleagues created a performance-based measure of risky behavior, called the "Balloon Analogue Risk Task" (BART; Lejuez et al. 2002). In this task, the participant can press a button to "pump up" computer images of balloons. Each pump of a balloon is rewarded, typically with money (e.g., 5 cents per pump), but if a balloon is pumped up too much, it explodes, and the participant receives no reward on that trial. The participant must decide at which point to press a "cash out" key to receive the reward on a given balloon before it explodes. Therefore, the desire for reward must be balanced against the potential risk of loss. Risk-taking on the task is typically measured as the mean number of pumps across trials for balloons that did not explode, which is referred to as the mean adjusted pumps.

Initial results from the BART have been promising. In the original validation study conducted in a predominately college student sample (Lejuez et al. 2002), mean adjusted pumps were positively correlated with self-reported impulsivity and sensation seeking, as well as with risky sexual behavior, substance use, and the average number of cigarettes smoked per day. Presumably risk-prone groups, such as individuals with substance abuse problems and conduct disorder, have also demonstrated more adjusted pumps than comparable control subjects (Crowley et al. 2006; Fernie et al. 2010; Holmes et al. 2009; Hopko et al. 2006; Hunt et al. 2005). In the two studies which have examined cigarette smoking and the BART, one in predominately Caucasian undergraduates (Lejuez et al. 2003) and one in predominately African American high school students (Lejuez et al. 2005), smokers had higher mean adjusted pumps than nonsmokers.

Although initial studies have suggested that smoking is associated with more adjusted pumps on the BART, it is unclear to what extent comorbidities of smoking may be playing a role in these findings. For example, abuse of alcohol and other substances is highly associated with smoking behavior in adolescence/young adulthood (Myers and Kelly 2006), and both alcohol (Fernie et al. 2010; Holmes et al. 2009; Lejuez et al. 2002) and drug abuse (Hopko et al. 2006; Lejuez et al. 2002) have also been associated with greater pumping on the BART. Only one of the aforementioned studies has examined comorbid drug use in smokers administered the BART (Lejuez et al. 2003), and it found that smoking was significantly and positively related to adjusted pumps after controlling for the number of different drug classes (e.g., marijuana and stimulants) the participants tried over the year before testing. However, this broad metric of substance abuse does not capture the severity of drug use in any particular drug class. In addition, none of the studies of smoking and the BART employed biological assays at the time of testing to determine whether participants had engaged in recent substance abuse. Further, these studies did not obtain information regarding current Axis I substance abuse or psychiatric disorders. This is potentially problematic as cigarette smoking has been associated with higher rates of psychiatric illness (Kalman et al. 2005), with some psychiatric disorders shown to be associated with BART performance (e.g., PTSD, Tull et al. 2009). Lastly, in a study designed to examine the effect of different psychotropic medications on impulsive and risky behavior (Acheson and de Wit 2008), adjusted pumps on the BART did not differ between a small group of adult smokers $(n=$ $10)$ and nonsmokers $(n=20)$ who did not have major Axis I psychiatric conditions and were verified to be abstinent from drugs through urinalyses and breathalyzer tests. While that study was limited by low statistical power, it raises the possibility that smoking may not be related to BART performance when participants with comorbidities are excluded.

Because the majority of the literature regarding smoking and the BART has not controlled for substance abuse and psychiatric conditions, it is uncertain whether risk-taking is uniquely related to smoking or would be more aptly characterized as a component of polysubstance abuse and/ or emotional dysfunction. To determine whether smoking per se is associated with a propensity for risk-taking, we conducted a study using the BART with young adult (ages 18 to 21$)$ smokers $(n=26)$ and non-smokers $(n=38)$, in which subjects with current psychiatric conditions, substance abuse and dependence (except nicotine), and recent drug use (i.e., positive urine tests for drugs or breathalyzer finding of recent alcohol use) were excluded. In order to examine the manner in which smokers and nonsmokers adapted to different risk contingencies on the BART, we included balloons with different risk probabilities and 
modeled pumping behavior on a trial-by-trial basis. Lastly, in order to more fully understand the construct assessed by the BART in this population, we examined the relationship between BART performance and measures of nicotine dependence, mood, impulsivity, estimated IQ, and demographic information. We hypothesized that smokers would exhibit more adjusted pumps than nonsmokers on the BART. In contrast to nonsmokers, we also expected smokers to display less aversion to pumping on the highrisk balloons when analyzed trial-by-trial (e.g., on the highrisk balloons, smokers would demonstrate flat pumping trajectories over time while nonsmokers would show decreasing trajectories over time).

\section{Method}

\section{Participants}

Participants consisted of 38 nonsmokers and 26 smokers (not seeking treatment) aged 18 to 21 . All participants were fluent in English and were administered the Structured Clinical Interview for the DSM-IV (SCID) for Axis I diagnosis (First et al. 1995) by a masters level clinician, as well as the self-report interview items from the KiddieSchedule for Affective Disorders and SchizophreniaPresent and Lifetime Version (K-SADS-PL, Kaufman et al. 1997) to assess attention deficit hyperactivity disorder (ADHD). Exclusion criteria were based on interview and physician-conducted history and physical examination. They were neurological disease (e.g., multiple sclerosis, head trauma with loss of consciousness $>5 \mathrm{~min}$ ), frank structural brain abnormalities on magnetic resonance imaging (MRI), systemic disease, cardiovascular disease, pulmonary disease, use of psychotropic medications, current Axis I psychiatric conditions or ADHD, and any current diagnosis of substance abuse or dependence. In addition, participants who tested positive for cocaine, marijuana, methamphetamine, benzodiazepines, or opiates in urinalysis during any assessment session were excluded, as were participants with positive results for alcohol on breathalyzer assessment. All smoking participants reported smoking daily and had urine cotinine levels $\geq 100 \mathrm{ng} / \mathrm{mL}$ and expired carbon monoxide (CO) levels $\geq 8 \mathrm{ppm}$ (SRNT 2002). ${ }^{1}$ Nonsmokers reported lifetime use of less than five cigarettes and had cotinine levels $\leq 30 \mathrm{ng} / \mathrm{mL}$ and expired $\mathrm{CO} \leq 4 \mathrm{ppm}$. Among participants who provided consent for the study (after initial phone screening), potential smokers were excluded due to positive urine tests $(n=20)$, current

\footnotetext{
${ }^{1}$ Six smokers had missing cotinine levels but still had expired $\mathrm{CO} \geq$ $8 \mathrm{ppm}$.
}

drug abuse/dependence $(n=22)$, psychiatric conditions or $\operatorname{ADHD}(n=5)$, and/or attrition, medical conditions, or other reasons $(n=9)$. Potential nonsmokers were excluded for psychiatric conditions or ADHD $(n=3)$, medical conditions $(n=2)$, positive drug tests $(n=1)$, excessive cotinine levels $(n=1)$, and/or attrition or other reasons $(n=13)$. Characteristics of the included participants are presented in Table 1 .

Measures

\section{Self-report measures}

Demographics form: a questionnaire that obtains demographic, employment, and educational information.

Drug use inventory: a self-report inventory that queries the duration and frequency of recent and lifetime substance use (e.g., alcohol, marijuana, cocaine, and opiates).

Cigarette dependence scale-12 (CDS-12 (Etter et al. 2003)): a 12-item questionnaire designed to assess the primary symptoms of nicotine dependence outlined in the DSM-IV.

Barratt impulsiveness scale-11 (BIS-11 (Patton et al. 1995)): a 30-item self-report questionnaire assessing impulsive personality traits.

Center for epidemiological studies-depression scale (CES-D (Radloff 1977)): a 20-item questionnaire developed from existing items on other well-validated measures of depression, assessing symptoms including depressive mood, guilt, feelings of worthlessness, psychomotor retardation, and sleep disturbance.

Spielberger trait anxiety scale (STAI-T (Spielberger et al. 1983)): a 20-item scale of trait anxiety assessing the disposition to be nervous, worried, tense, and insecure.

\section{Behavioral measures}

Wechsler abbreviated scale of intelligence (WASI (Wechsler 1999)): a brief measure of intelligence. The Vocabulary subtest was used to estimate verbal IQ, and the Matrix Reasoning subtest was used to estimate nonverbal IQ. Raw scores were converted to T-scores using age-based normative data.

Delay discounting test (DDT (Kirby et al. 1999)): a decision-making task in which participants must indicate their preference for one of two hypothetical options across 27 trials. One option consists of receiving an amount of money immediately, and the other option consists of receiving a larger amount of money at a later point in time. The discrepancy between the amounts of money and the duration of the delay period ( 7 to 186 days) is varied across trials. 
Table 1 Characteristics of research participants
Where appropriate, values are means \pm SD. Verbal IQ estimate $=$ WASI Vocabulary subtest; nonverbal IQ estimate= WASI Matrix Reasoning subtest $* p<.05 ; * * p<.01 ; * * * 0.05 \geq$ $p \leq 0.06$, non-significant trend

${ }^{a}$ Larger (toward positive) scores indicate greater discounting

${ }^{\mathrm{b}}$ One control subject used Vicodin on one of the last 30 days

\begin{tabular}{|c|c|c|c|}
\hline & Statistical test & Nonsmokers $(n=38)$ & Smokers $(n=26)$ \\
\hline \multicolumn{4}{|l|}{ Demographics } \\
\hline Age (years) & $t(62)=-1.18$ & $19.8 \pm 1.1$ & $20.2 \pm 1.2$ \\
\hline Education (years) & $t(62)=0.81$ & $14.0 \pm 1.2$ & $13.8 \pm 1.2$ \\
\hline Mother's education (years) & $t(60)=-0.81$ & $13.6 \pm 2.8$ & $14.2 \pm 2.3$ \\
\hline Father's education (years) & $t(59)=0.75$ & $14.6 \pm 2.9$ & $14.0 \pm 2.9$ \\
\hline Verbal IQ estimate (T-score) & $t(60)=-0.56$ & $59.2 \pm 9.5$ & $60.4 \pm 6.6$ \\
\hline Nonverbal IQ estimate (T-score) & $t(61)=0.07$ & $58.1 \pm 5.2$ & $58.0 \pm 6.1$ \\
\hline Not currently in school & $\chi^{2}(1, N=64)=4.24$ & 3 & $7 *$ \\
\hline Currently employed & $\chi^{2}(1, N=63)=0.01$ & 18 & 13 \\
\hline Median income, city of residence $(\$)$ & $t(55)=0.25$ & $61,276 \pm 24,264$ & $59,866 \pm 16,954$ \\
\hline Ethnicity & $\chi^{2}(4, N=64)=6.06$ & & \\
\hline Caucasian & & 10 & 10 \\
\hline African American & & 5 & 0 \\
\hline Hispanic & & 6 & 6 \\
\hline Asian/Pacific Islander & & 12 & 9 \\
\hline Other & & 5 & 1 \\
\hline Gender (male/female) & $\chi^{2}(1, N=64)=0.16$ & $18 / 20$ & $11 / 15$ \\
\hline \multicolumn{4}{|l|}{ Mood/personality } \\
\hline Depression (CES-D) & $t(62)=-0.69$ & $9.6 \pm 5.4$ & $10.7 \pm 6.8$ \\
\hline Trait anxiety (STAI-T) & $t(62)=0.27$ & $36.8 \pm 7.5$ & $36.2 \pm 10.9$ \\
\hline Barratt impulsiveness total score & $t(61)=-1.88$ & $59.4 \pm 9.0$ & $63.7 \pm 8.8 * * *$ \\
\hline Delay discounting total $k$ value $(\log )^{\mathrm{a}}$ & $t(61)=-2.90$ & $-4.9 \pm 1.8$ & $-3.8 \pm 1.0^{*}$ \\
\hline \multicolumn{4}{|l|}{ Substance use } \\
\hline Days drank beer last 30 days & $t(62)=-3.81$ & $1.3 \pm 1.9$ & $4.0 \pm 3.8 * *$ \\
\hline Days drank liquor last 30 days & $t(62)=-3.18$ & $1.1 \pm 1.6$ & $3.4 \pm 4.1 * *$ \\
\hline Days used marijuana last 30 days & $t(62)=-1.99$ & $0.13 \pm 0.4$ & $0.50 \pm 1.03 * * *$ \\
\hline Days used opiates last 30 days ${ }^{\mathrm{b}}$ & - & $0.03 \pm 0.16$ & - \\
\hline Days used cocaine last 30 days & - & - & - \\
\hline Days used amphetamine last 30 days & - & - & - \\
\hline Days used hallucinogens last 30 days & - & - & - \\
\hline Cigarettes smoked per day & - & - & $7.8 \pm 3.7$ \\
\hline Cigarette dependence scale & - & - & $38.1 \pm 7.2$ \\
\hline
\end{tabular}

A hyperbolic statistical function can be fit to each trial to estimate the point at which an individual would be indifferent between the two options. The discounting of delayed rewards is taken to be the indifference point, or total $k$ value, which is most consistent with the participant's selections across the task (for details, see Kirby et al. (1999)). Discounting $k$ values were normalized with the natural log function in accordance with the procedure of Kirby et al. (1999). Although the DDT can be done with paper and pencil, we administered the task on a computer, one trial at a time.

Balloon analogue risk task (BART; modeled after Lejuez et al. (2002)): a computerized task of risky decision-making in which participants press a key to inflate balloons. Each pump (key press) of a balloon earns the participant 2 cents, but if balloons are pumped up too much, they explode, and no money is earned on that trial. On each trial, the participant has to hit a "cash out" key prior to balloon explosion in order to place that trial's money into a cumulative bank. Balloons are colored blue and red, with 40 balloons presented in total (20 of each color presented in random sequence). Red balloons are relatively "high risk"; they have a $1 / 32$ probability of exploding on the first pump and an incrementally ascending probability of explosion with each successive pump (1/31 on the second pump, $1 / 30$ on the third pump, etc.). Blue balloons are "low risk," with a $1 / 128$ probability of exploding on the first pump (1/127 on the second pump, etc.). The primary summary variable from the 
BART is the mean number of pumps across trials in which the balloon did not explode, referred to as mean adjusted pumps. Adjusted pumps are considered preferable to absolute pumps because explosions restrict the range of risk behavior (for evidence of the bias associated with absolute pumps, see Pleskac et al. (2008)). Participants received their earnings in cash. Based on the probabilities of explosion, earnings on the high-risk balloon can be maximized by pumping an average of 16 times per balloon, while earnings on the low-risk balloon can be maximized by pumping 64 times per balloon (Lejuez et al. 2002).

Instructions for the BART were similar to those implemented in the original validation study of the instrument (Lejuez et al. 2002), except that Lejuez and colleagues used three balloon colors with three initial risk probabilities $(1 / 128,1 / 32$, and $1 / 8)$ and informed the participants that "the explosion point varies across balloons." Because we used two balloon colors and wished to reduce the effect of learning on risk behavior (e.g., see Wallsten et al. 2005), we informed the participants beforehand that "one [balloon] color is more likely to explode than the other." For consideration of other slight differences between our BART and the versions used in Lejuez' studies (Lejuez et al. 2002, 2003, 2005), please see the "Discussion" section.

\section{Procedure}

Participants were recruited from the greater Los Angeles area via newspaper and Internet advertisements. Potential participants were preliminarily screened for inclusion/ exclusion on the telephone, and those that passed the initial interview were invited for in-person intake screening with the SCID, K-SADS-PL, medical examination, and selfreport questionnaires. At intake, participants first received a detailed description of the protocol and provided written informed consent following the guidelines of the UCLA Office for Protection of Research Subjects. Qualified participants were subsequently scheduled to complete the behavioral measures on a separate testing day, as well as a structural MRI scan and cognitive tests not covered in this manuscript. Urinalysis and breathalyzer tests were performed on the intake and behavioral assessment days to assure drug and alcohol abstinence. Smoking participants were allowed to take smoking breaks as needed during both sessions so that they would not be in a state of nicotine withdrawal (e.g., Domier et al. 2007). Because the current study was part of a larger study on the neural systems of risk-taking in adolescent smokers, participants frequently underwent structural MRI on the same day as the BART, as well as up to two functional MRI scans on subsequent testing days. Participants were paid $\$ 50$ for the intake session, \$30 for the behavioral session, up to $\$ 20$ based on their BART performance, and also received payment for MRI scans as appropriate.

\section{Statistical analyses}

Statistical analyses were organized into four main sections: (1) sample characterization analyses, in which demographic and mood/personality differences between smokers and nonsmokers were analyzed with $t$ tests or Chi-square analyses, as appropriate; (2) BART summary score analyses, in which smokers and nonsmokers were compared with twosample $t$ tests on the primary BART global variable, mean adjusted pumps, as well as on secondary BART composite variables such as absolute pumps, number of explosions, and variability in pumps; (3) BART trajectory analyses, in which the manner in which smokers and nonsmokers adapted to the task across trials was analyzed with general linear mixed models (GLMMs). We were particularly interested in how participants adapted to the different balloon risk types across trials; and (4) exploratory analyses, in which demographic and questionnaire data were related to BART performance using the GLMM. The purpose of these analyses was to determine which study variables were related to BART performance, both overall and on a trial-by-trial basis, as a means to better understand the construct assessed by the task. It should be noted that these analyses were not hypothesis-driven and were conducted at an uncorrected statistical threshold $(p<.05)$.

\section{Results}

Sample characterization analyses

The smokers and nonsmokers did not differ significantly in age, gender, ethnicity, years of education, IQ estimates, years of mother's or father's education, likelihood of being employed, median income of city of residence, depression scores, or trait anxiety $\left(p^{\prime} s>.05\right)$. However, the smokers discounted delayed rewards to a greater extent than nonsmokers ( $\log$ total $k$ value, $t(61)=-2.90, p=.012$ ) and trended toward higher levels of total impulsiveness on the BIS-11 $(t(61)=-1.88, p=.064)$. Inspection of the BIS-11 subscales indicated that this effect was primarily driven by higher levels of motor impulsivity in the smoking group $\left(t(61)=-2.83, p=.006\right.$; all other subscales $\left.p^{\prime} \mathrm{s}>.15\right)$. In addition, the smokers consumed beer $(t(62)=-3.81, p=.000)$, liquor $(t(62)=-3.18, p=.002)$, and marijuana $(t(62)=-1.99$, $p=.051$ ) on more of the previous 30 days than the nonsmokers, although overall usage in both groups was mild (mean of less than 5 days of the last 30 for each 
substance). The smokers were also less likely than nonsmokers to be currently in school $\left(\chi^{2}(1, N=64)=4.24, p=.039\right)$. Although smokers and nonsmokers did not differ significantly in ethnicity $\left(\chi^{2}(4, N=64)=6.06, p=.20\right)$, it should be noted that the nonsmoking group had African American subjects $(n=5)$, while the smoking group did not, and it also had more "other" participants than the smoking group (5 vs. 1 , respectively).

\section{BART summary score analyses}

The smokers and nonsmokers did not differ significantly on either the primary composite outcome measure, mean adjusted pumps, or on any of the subset measures: mean "high-risk balloon" adjusted pumps, mean "low-risk balloon" adjusted pumps, and mean high- or low-risk explosions $(p$ 's $>.30)$. The smokers and nonsmokers also did not differ on the mean number of adjusted pumps on the high- and low-risk balloons on trials which immediately followed an explosion (i.e., trials in which perceived risk may be heightened, $p^{\prime} \mathbf{s}>.30$ ), nor did they differ on a measure of pumping variability (see Jentsch et al. 2010), in which the variance of adjusted pumps was divided by the mean adjusted pumps for each balloon risk type ( $\left.p^{\prime} \mathbf{s}>.20\right)$. We note that not only was there no evidence of an overall average difference in risky behavior between the groups but that in fact the mean adjusted pumps and explosions were typically lower (nonsignificantly) in the smokers than the nonsmokers (identical results were obtained if mean unadjusted pumps were considered: $t(62)=0.87, p>.05$; smokers 15.8 \pm 6.2 ; nonsmokers 17.3 \pm 7.3 ). Thus, the absence of riskier behavior in the smokers was not simply a result of low power. The above results were unchanged when the group comparisons were adjusted for substance use in the last 30 days (beer, liquor, and marijuana), current school attendance, delay discounting, motor impulsivity, and ethnicity. Performance of the smokers and nonsmokers on the BART summary variables is presented in Table 2 . Correlations among the BART summary variables and the other primary variables of interest are displayed in Table 3 .

\section{BART trajectory analyses}

In order to examine the manner in which the smokers and nonsmokers adapted to the BART across balloon trials, we fit a GLMM with group (smoker vs. nonsmoker), risk condition (high- vs. low-risk balloons), balloon trial (1-20 within each balloon risk condition, treated as continuous), and all possible interactions thereof as independent variables and pumps per trial (on non-exploded balloons) as the dependent variable. The GLMM is mathematically equivalent to a repeated measures model, but the fitting procedure automatically allows for the missing data produced by excluded explosion trials. The resulting estimates are valid and unbiased to missing observations provided that the values are missing at random or the factors associated with the pattern of missingness are accounted for in the model (Fitzmaurice et al. 2004; Laird 1988; Little and Rubin 2002). That is the case here since the balloon explosions are driven by the underlying probability of popping and the subjects' pumping profiles, both of which are fully modeled by the observed trajectories. The three-way interaction of group, risk condition, and trial was not significant $(F(1,1,657)=0.94, p=.33)$, indicating that the relative amount of adaptation across trials to the low- and high-risk conditions did not differ between smokers and nonsmokers. However, there were significant two-way interactions between risk condition and balloon trial $(F(1,1,657)=24.43, p=.000)$ and group and balloon trial $(F(1,1,657)=15.83, p=.000)$. These interactions were driven by the fact that on the low-risk balloons, the nonsmokers appeared to increase their pumping across trials, while the smokers remained relatively stable, whereas on the high-risk balloons, the nonsmokers kept their pumping behavior relatively stable, while the smokers appeared to become more risk averse (see Fig. 1). There was also a significant main effect of risk condition $(F(1$, $1,657)=74.51, p=.000)$, but all other main effects and interactions were nonsignificant $(p>.05)$. The potential confounding effects of substance use in the last 30 days (beer, liquor, and marijuana), school attendance, delay discounting, BIS motor scores, and ethnicity were evaluated by adding their main effects and two-way interaction terms (with group, risk condition, and balloon trial) to the original model. The pattern of results reported above was unchanged. In order to determine whether the pumping trajectories described above were increasing or decreasing (i.e., not flat), we tested whether the parameters corresponding to the slopes for balloon trial were significantly different from 0 for each of the four group/risk condition combinations. As expected, the nonsmokers significantly increased their pumping over trials on the low-risk balloon $(\beta=.47, p<.000)$, and the smokers significantly decreased their pumping over trials on the highrisk balloon $(\beta=-.25, p=.011)$. In contrast, the trajectories for nonsmokers on the high-risk balloon $(\beta=-.01, p=.96)$ and the smokers on the low-risk balloon $(\beta=.07, p=.34)$ were not significantly different from 0 .

\section{Exploratory analyses}

In order to explore possible relationships between BART performance and the demographic, intellectual, mood, substance use, and impulsivity variables shown in Table 1, we ran GLMM models as above, but added main effects for each Table 1 variable and the corresponding interactions with balloon trial. This allowed us to determine whether 
Table 2 Comparison of BART variables between smokers and nonsmokers

\begin{tabular}{|c|c|c|c|c|}
\hline BART variable & Statistical test & Nonsmokers & Smokers & $p$ value \\
\hline Adjusted pumps & $t(62)=0.97$ & $20.3 \pm 9.7$ & $18.1 \pm 7.8$ & 0.34 \\
\hline Low-risk adjusted pumps & $t(62)=1.02$ & $26.6 \pm 15.6$ & $22.9 \pm 12.8$ & 0.31 \\
\hline High-risk adjusted pumps & $t(62)=0.28$ & $11.2 \pm 4.0$ & $10.9 \pm 4.0$ & 0.78 \\
\hline Low-risk explosions & $t(62)=0.93$ & $4.2 \pm 2.4$ & $3.6 \pm 2.2$ & 0.35 \\
\hline High-risk explosions & $t(62)=-0.63$ & $8.9 \pm 2.6$ & $9.4 \pm 2.6$ & 0.48 \\
\hline Low-risk pumps after an explosion & $t(62)=1.02$ & $24.9 \pm 15.3$ & $21.1 \pm 13.1$ & 0.31 \\
\hline High-risk pumps after an explosion & $t(62)=0.30$ & $10.6 \pm 4.4$ & $10.3 \pm 4.1$ & 0.77 \\
\hline Variance/mean low-risk pumps ${ }^{\mathrm{a}}$ & $t(62)=0.64$ & $2.7 \pm 2.7$ & $2.3 \pm 2.2$ & 0.52 \\
\hline Variance/mean high-risk pumps ${ }^{\mathrm{a}}$ & $t(62)=0.28$ & $1.5 \pm 1.2$ & $1.9 \pm 1.5$ & 0.21 \\
\hline
\end{tabular}

Values are means \pm SD. Low risk refers to blue balloons with a $1 / 128$ starting probability of explosion. High risk refers to red balloons with a $1 / 32$ starting probability of explosion

${ }^{a}$ Variance/mean is the variance of adjusted pumps divided by the mean number of adjusted pumps, which has been provided for each balloon risk type (see Jentsch et al. 2010)

each Table 1 variable had a significant main effect on BART performance, or if it contributed to pumping trajectories over time (i.e., variable by trial interaction). The variables from Table 1 were evaluated one at a time along with the independent variables previously employed in the BART trajectory analyses (i.e., group, risk condition, trial, and the group by trial interaction). The three-way interaction (group $\times$ risk condition $\times$ trial) and the two-way group by risk condition interaction previously entered in the original BART trajectory analyses were not included here because they did not significantly contribute to the original model fit.

Variable by balloon trial interactions The above analyses produced a significant variable by balloon trial interaction for ethnicity $(F(4,1,655.1)=2.82, p=.024)$. Performance plots revealed that African American subjects increased

Table 3 Intercorrelations among summary BART variables and other variables of interest

\begin{tabular}{|c|c|c|c|c|c|c|c|c|c|c|c|c|}
\hline Variable & HRP & LRP & HREx & LREx & Age & Edu & VIQ & PIQ & BIS & DDT & Dep & Anx \\
\hline High-risk pumps (HRP) & - & & & & & & & & & & & \\
\hline Low-risk pumps (LRP) & $0.30^{*}$ & - & & & & & & & & & & \\
\hline $\begin{array}{l}\text { High-risk explosions } \\
\text { (HREx) }\end{array}$ & $0.56^{* *}$ & $0.45^{* *}$ & - & & & & & & & & & \\
\hline $\begin{array}{l}\text { Low-risk explosions } \\
\text { (LREx) }\end{array}$ & $0.30^{*}$ & $0.78^{* *}$ & $0.42 * *$ & - & & & & & & & & \\
\hline Age & -0.02 & 0.05 & 0.18 & 0.01 & - & & & & & & & \\
\hline Education (Edu) & 0.11 & $0.27 *$ & 0.22 & $0.28 *$ & $0.74 * *$ & - & & & & & & \\
\hline Verbal IQ (VIQ) & $0.28^{*}$ & 0.18 & 0.10 & 0.13 & -0.01 & 0.04 & - & & & & & \\
\hline Performance IQ (PIQ) & 0.23 & 0.23 & 0.06 & $0.32 *$ & 0.17 & $0.30 *$ & 0.16 & - & & & & \\
\hline BIS & -0.05 & -0.05 & 0.07 & 0.01 & 0.08 & 0.03 & 0.13 & 0.09 & - & & & \\
\hline $\begin{array}{l}\text { Delay discounting task } \\
\text { (DDT) }\end{array}$ & -0.19 & -0.01 & -0.06 & -0.13 & 0.11 & -0.02 & $-0.40 * *$ & -0.01 & -0.10 & - & & \\
\hline Depression (Dep) & -0.16 & 0.07 & -0.00 & 0.05 & 0.12 & 0.15 & 0.04 & -0.13 & $0.47 * *$ & 0.15 & - & \\
\hline Anxiety (Anx) & -0.06 & 0.09 & 0.06 & 0.12 & 0.07 & 0.11 & 0.12 & -0.09 & $0.36^{* *}$ & 0.01 & $0.70 * *$ & - \\
\hline
\end{tabular}

High-risk pumps $(\mathrm{HRP})=$ mean pumps on balloons with $1 / 32$ starting probability of explosion; Low-risk pumps $($ LRP $)=$ mean pumps on balloons with 1/128 starting probability of explosion; High-risk explosions (HREx)=number of explosions on high-risk balloons; Low-risk explosions $($ LREx $)=$ number of explosions on low-risk balloons; Age=age in years; Education $($ Edu $)=$ education in years; Verbal IQ $($ VIQ $)=$ verbal IQ estimated by Vocabulary subtest on Wechsler Abbreviated Test of Intelligence (WASI); Performance IQ (PIQ)=nonverbal IQ estimated by Matrix Reasoning test on WASI; BIS=Barratt Impulsiveness Scale-11; Delay Discounting Task (DDT) $=\operatorname{logged}$ total $k$ value; Depression=Center for Epidemiological Studies-Depression Scale (CES-D); Anxiety=Spielberger Trait Anxiety Scale (STAI-T)

${ }^{*} p<.05$ (uncorrected); ${ }^{*} p<.01$ (uncorrected) 


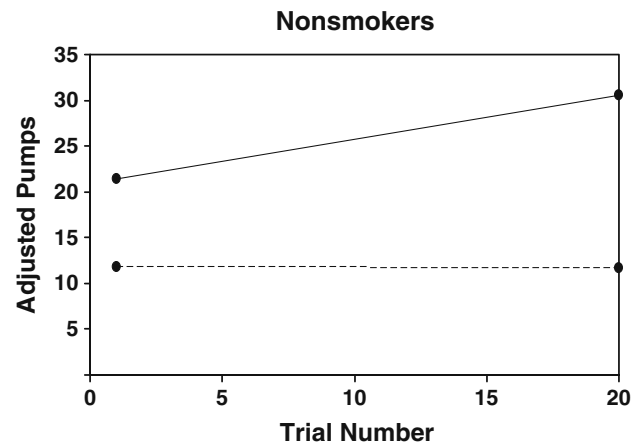

Fig. 1 Trajectories of adjusted pumps by smokers and nonsmokers across Balloon Trials of the BART. Lines represent regression lines derived from the general linear mixed model (GLMM). Low-risk balloons $=1 / 128$ starting probability of explosion. High-risk balloons $=$

their pumping trajectories across trials to a greater degree than other ethnicities. However, because all African American participants were nonsmokers, the implications of this finding are unclear. A trend for significance was also found in the interaction between currently being employed and balloon trial $(F(1,1,629.4)=3.69, p=.055)$, with participants who were employed increasing their pumping trajectories across trials to a greater extent than unemployed subjects. No other significant interactions were observed between balloon trial and any of the Table 1 variables $\left(p^{\prime} \mathbf{s}>.05\right)$. Also, it should be noted that adding the main effects of ethnicity and employment and their interactions with balloon trial to the original BART trajectory model did not change the significance of the aforementioned interactions between smoking group and balloon trial $(p<.001)$.

Variable main effects We also found a significant main effect for years of education $(F(1,79.7)=6.55, p=.012)$ and a trend for a main effect of nonverbal IQ $(F(1,78.5)=3.92$, $p=.051)$. Both years of education and nonverbal IQ were positively related to adjusted pumps. (Since education and nonverbal IQ are likely to be correlated, we also ran a joint model with both education and nonverbal IQ and their interactions with balloon trial; education remained significant $(F(1,77.2)=4.11, p=.046)$, while nonverbal IQ did not $(F(1,77.5)=1.73, p=.19)$.) Although there was no main effect for the logged delay discounting $k$ value in our Table 1 variable analyses, we did find a significant main effect for the raw $k$ value $(F(1,76.0)=5.48, p=.022)$. Inspection of scatterplots showed that the negative relationship between the raw $k$ value and adjusted pumps $(r=-.33, p=.009)$ was driven by five outlier subjects (three smokers, two nonsmokers) with very high delay discounting scores ( $>2$ standard deviations above the mean) and low pumps, thus normalizing these high scores with the log function eliminated the main effect. No other

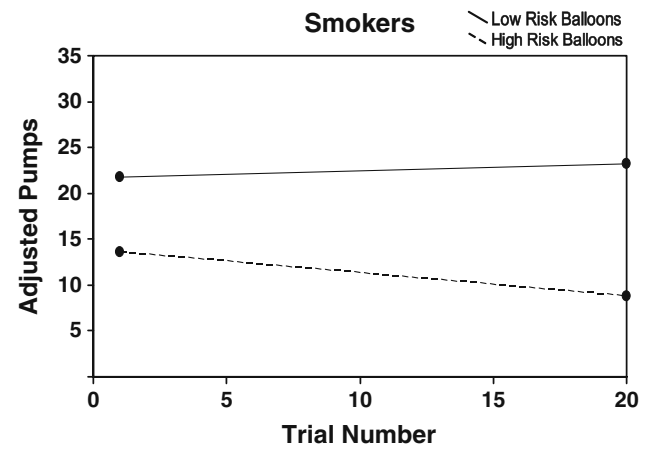

1/32 starting probability of explosion. Low-risk and high-risk balloons were presented in random order in the 40 balloon trials; thus, Trial Number reflects the order within each balloon type, not the overall order across balloon types

main effects were observed between Table 1 variables and BART performance $\left(p^{\prime} \mathrm{s}>.05\right)$. Also, within the smoking group only, measures of nicotine dependence and cigarettes smoked per day were unrelated to BART performance (i.e., there were no main effects or interactions with balloon trial).

\section{Discussion}

Contrary to previous research on the BART in adolescent and young adult smokers (Lejuez et al. 2002, 2003, 2005), we did not find smokers to exhibit greater risk-taking than nonsmokers on standard BART composite indices (e.g., pumps, explosions, pumps after explosions, and variability). In fact, in analyses at the balloon trial level, the smokers and nonsmokers showed differential adaptation in risk behavior, with the nonsmokers exhibiting progressively more pumping relative to the smokers over the course of the task, regardless of the type of risk condition. Both groups pumped more overall on the low-risk balloons than the high-risk balloons. The group effects we observed were not attributable to demographic, substance abuse, or mood variables.

Because our study employed more rigorous exclusion of drug use and psychiatric comorbidities than previous studies (Lejuez et al. 2002, 2003, 2005), the relatively limited risky behavior exhibited by our smoking group may have been attributable to the removal of those with potentially risk-prone comorbidities; in other words, young adult smokers who do not have substance abuse problems or psychiatric comorbidities may not engage in risky behaviors. However, we find this explanation unlikely, as the absence of drug abuse and psychiatric diagnoses from the smoking group would not explain why the smokers displayed a significantly more risk averse pumping strategy 
than the nonsmokers over the course of the BART assessment. Further, the smoking group otherwise performed in a manner that would imply higher rates of risky or impulsive behavior than nonsmokers. For example, the smokers had a greater tendency to discount delayed rewards than the nonsmokers, and they had higher levels of motor impulsivity. In addition, despite the removal of those with substance abuse diagnoses, the smokers endorsed using alcohol on more of the previous 30 days than nonsmokers, as well as a trend towards more use of marijuana $(p=.05)$. Nonetheless, given the exclusion of drug abuse and psychiatric comorbidities from our smoking group, our BART findings may only be generalizable to smokers who do not have comorbid conditions.

Although divergent from some previous research (Lejuez et al. 2003, 2005), the absence of significant differences in mean adjusted pumps between smokers and nonsmokers is similar to the results of Acheson and de Wit (2008), who also did not find a group effect for adjusted pumps in a small sample of smokers $(n=10)$ and nonsmokers $(n=20)$ in a study assessing the effects of medication on the BART. Similarly, not all research has suggested that adjusted pumps on the BART are positively associated with maladaptive traits or psychopathology. For example, in a recent investigation, Ray and Ashenhurst (2010) found that BART adjusted pumps and post-explosion pumps were negatively associated with alcohol problem behaviors in a sample of 51 participants with alcohol use disorders. Humphreys and Lee (manuscript under editorial review) similarly found that, after controlling for symptoms of oppositional defiance, ADHD symptoms were negatively related to adjusted pumps and post-explosion pumps in a large sample $(n=111)$ of ethnically diverse children aged 6 to 9 .

Additional insight into the nature of our findings was provided by examination of relationships between BART performance and measures of mood, impulsivity, and demographic characteristics. Adjusted pumps on the BART were positively related to years of education, with a trend for a positive relationship between adjusted pumps and estimated nonverbal IQ $(p=.051)$. A small subset $(n=5)$ of individuals with very high delay discounting scores also had low levels of pumping on the BART. This perhaps suggests that these individuals preferred the immediate reward of "cashing in" early, rather than persisting with pumping to earn, or lose, greater sums of money.

With respect to pumping trajectories, employed participants tended to increase their pumping over trials to a greater extent than those who were unemployed $(p=.055)$. While perhaps those without a job were more risk averse because they perceived the money earned on the BART to be of more value than those who were employed, we did not find a relationship between BART performance and the median income of the city in which participants resided (some unemployed subjects were students receiving money from other sources), nor did we find a relationship between BART performance and parental education levels, a common proxy for socioeconomic background (Bradley and Corwyn 2002). Importantly, controlling for the effect of employment did not alter the trajectory differences found between smokers and nonsmokers on the BART.

Our overall findings suggest that greater pumping on the BART was related to positive traits (e.g., nonsmoking, employment, years of education, and higher IQ). Although divergent from studies of other patient groups, these results are consistent recent studies demonstrating negative relationships between pumping and dysfunctional symptoms in other populations (Humphreys and Lee, manuscript under editorial review; Ray and Ashenhurst 2010). It is also important to note that, as a result of their pumping trajectories, nonsmokers made a nonsignificantly greater amount of money on the task than smokers. Further, neither the smokers or the nonsmokers exhibited mean adjusted pumps that came close to exceeding the optimal reward/risk ratio for either balloon risk condition ("low-risk" balloons, smoker mean $=22.9$; nonsmoker mean $=26.6$; optimal mean= 64 (Lejuez et al. 2002); "high-risk" balloons, smoker mean= 11.2; nonsmoker mean $=10.9$; optimal mean $=16$ (Lejuez et al. 2002)). This is further exemplified by the strong positive correlation between explosions and total money earned on the BART $(r=.63, p=.000)$. In other words, participants who pumped more on the task generally made more money than those who were more conservative.

These findings indicate that, at the levels of risk-taking demonstrated here, pumping was generally adaptive, not maladaptive. This is a common occurrence in studies of the BART, in which participants rarely exceed the optimal level of pumps, regardless of the population under investigation (see Lejuez et al. 2002; Pleskac et al. 2008). Thus, although excessive pumping on the BART has been associated with maladaptive traits, low levels of pumping may also be indicative of poor decision-making. Because risks are present to varying degrees in the day-to-day activity of living, these data serve as a reminder that the ultimate adaptiveness of a potentially risky action is based not only on the consequences of acting but also on the consequences of failing to act. Further, since the pumping trajectories exhibited by nonsmokers were associated (nonsignificantly) with earning more money on the task, it may be a misnomer to characterize their behavior as "riskier" than the smokers. Rather, relative to the smokers, their behavior may be better described as reward sensitive.

Although differences between our BART and the BART(s) used by Lejuez and colleagues might account for the differences in results between the studies, we generally find this explanation inadequate. In the original BART validation 
study, three balloon colors were used with three different initial explosion probabilities: $1 / 8,1 / 32$, and 1/128. The balloons with higher explosion probabilities were primarily included as a means to ensure that some balloons exploded (Lejuez et al. 2002), and adjusted pumps on just the lowest probability balloon $(1 / 128)$ was used as the primary dependent variable in this and subsequent studies (Lejuez et al. 2003, 2005). Our study included two probabilities of explosion: $1 / 32$ ("high risk") and 1/128 ("low risk"). The higher risk condition (1/32 initial probability) was sufficient to ensure explosions, and thus, we do not suspect that differences in explosion probability account for the differences in our results. Further, differences between our study and previous studies are also unlikely to be attributable to the number of balloon trials used. We found significant differences in pumping trajectories using a smaller number of trials: 40 total balloons (20 per condition) vs. previous studies with 90 balloons (30 per color, Lejuez et al. 2002) and 30 balloons (one color, Lejuez et al. 2003, 2005), respectively, the latter of which reported relatively parallel trajectories for smokers and nonsmokers.

Because we used only two balloon risk conditions and wished to reduce the effect of learning on pumping behavior, we informed participants beforehand that "one [balloon] color is more likely to explode than the other." While it is possible that nonsmokers took advantage of this information to a greater extent than smokers, it is clear that both smokers and nonsmokers discriminated between the low-risk and high-risk balloons almost from the beginning of the task (see Fig. 1). Moreover, these instructions would not explain why smokers displayed risk averse pumping trajectories compared to the nonsmokers on the high-risk balloon type.

Previous studies examining the relationship between smoking and the BART have varied in the rewards used for pumping behavior: the original validation study gave participants 5 cents per pump (Lejuez et al. 2002), while later studies rewarded participants for total points on the task with movie tickets, gift cards, or a flat payment of $\$ 10$ (Lejuez et al. 2003,2005 ). These values are grossly similar to our study, in which participants received 2 cents per pump, earning them between $\$ 1.90$ and $\$ 20.00$ on the task. In addition, previous research suggests that paying only 1 cent per pump is a sufficient incentive to demonstrate greater pumping in adolescents with conduct problems and substance abuse compared to control subjects (Crowley et al. 2006).

\section{Conclusions and limitations}

As demonstrated through our recruiting process, smoking behavior in young adults is often comorbid with diagnoses of substance abuse and dependence. As such, generalizations regarding the behavior of "smokers" must be done with caution if substance abuse and dependence have not been properly controlled. The rigorous exclusion of substance abuse and psychiatric disorders in the current study served as a strength in the experimental isolation of smoking-specific effects. However, these exclusions may also reduce the generalizability of our findings to smokers in the community, in which comorbidities are commonplace. Indeed, our BART results may only generalize to smokers who do not have comorbidities. It should also be noted that our sample sizes were relatively small, and this also limits the potential generalizability of our results. Nonetheless, our data suggest that, although smoking is a clear health risk behavior, in certain circumstances, young adult smokers may take less adaptive risks than their nonsmoking counterparts.

Funding support The research described in this article was funded, in part, by a grant from Philip Morris USA. Additional funding was provided by NIH grant K23 DA027734 (Dean) and an endowment from the Thomas P. and Katherine K. Pike Chair in Addiction Studies and by a generous gift from the Marjorie M. Greene Trust. None of the sponsors had any involvement with the design, collection, analysis, or interpretation of data, writing the manuscript, or the decision to submit the manuscript for publication. The authors declare no conflicts of interest.

Open Access This article is distributed under the terms of the Creative Commons Attribution Noncommercial License which permits any noncommercial use, distribution, and reproduction in any medium, provided the original author(s) and source are credited.

\section{References}

Acheson A, de Wit H (2008) Bupropion improves attention but does not affect impulsive behavior in healthy young adults. Exp Clin Psychopharmacol 16:113-123

Bradley RH, Corwyn RF (2002) Socioeconomic status and child development. Annu Rev Psychol 53:371-399

CDC (2002) Annual smoking-attributable mortality, years of potential life lost, and economic costs-United States, 1995-1999. MMWR Morb Mortal Wkly Rep 51:300-303

CDC (2005) Annual smoking-attributable mortality, years of potential life lost, and productivity losses-United States, 1997-2001. MMWR Morb Mortal Wkly Rep 54:625-628

Crowley TJ, Raymond KM, Mikulich-Gilbertson SK, Thompson LL, Lejuez CW (2006) A risk-taking "set" in a novel task among adolescents with serious conduct and substance problems. J Am Acad Child Adolesc Psychiatry 45:175-183

DiFranza JR, Winters TH, Goldberg RJ, Cirillo L, Biliouris T (1986) The relationship of smoking to motor vehicle accidents and traffic violations. NY State J Med 86:464-467

Domier CP, Monterosso JR, Brody AL, Simon SL, Mendrek A, Olmstead R, Jarvik ME, Cohen MS, London ED (2007) Effects of cigarette smoking and abstinence on Stroop task performance. Psychopharmacology 195:1-9

Eaton DK, Kann L, Kinchen S, Shanklin S, Ross J, Hawkins J, Harris WA, Lowry R, McManus T, Chyen D, Lim C, Whittle L, Brener 
ND, Wechsler H (2010) Youth risk behavior surveillanceUnited States, 2009. MMWR Surveill Summ 59:1-142

Etter JF, Le Houezec J, Perneger TV (2003) A self-administered questionnaire to measure dependence on cigarettes: the cigarette dependence scale. Neuropsychopharmacology 28:359-370

Fernie G, Cole JC, Goudie AJ, Field M (2010) Risk-taking but not response inhibition or delay discounting predict alcohol consumption in social drinkers. Drug Alcohol Depend 112:54-61

First MB, Spitzer RL, Gibbon M, Williams JBW (1995) The structured clinical interview for DSM-IV Axis I disorders (SCID-IP). American Psychiatric Press, Washington

Fitzgerald JL, Mulford HA (1987) Self-report validity issues. J Stud Alcohol 48:207-211

Fitzmaurice GM, Laird NM, Ware JH (2004) Applied longitudinal analysis. Wiley, Hoboken

Hanna EZ, Grant BF (1999) Parallels to early onset alcohol use in the relationship of early onset smoking with drug use and DSM-IV drug and depressive disorders: findings from the National Longitudinal Epidemiologic Survey. Alcohol Clin Exp Res 23:513-522

Hersch J, Viscusi WK (1998) Smoking and other risky behaviors. J Drug Issues 28:645-661

Holmes KM, Bearden CE, Barguil M, Fonseca M, Serap Monkul E, Nery FG, Soares JC, Mintz J, Glahn DC (2009) Conceptualizing impulsivity and risk taking in bipolar disorder: importance of history of alcohol abuse. Bipolar Disord 11:33-40

Hopko DR, Lejuez CW, Daughters SB, Aklin WM, Osborne A, Simmons BL, Strong DR (2006) Construct validity of the Balloon Analogue Risk Task (BART): relationship with MDMA use by inner-city drug users in residential treatment. J Psychopathol Behav Assess 28:95-101

Hunt MK, Hopko DR, Bare R, Lejuez CW, Robinson EV (2005) Construct validity of the balloon analog risk task (BART): associations with psychopathy and impulsivity. Assessment $12: 416-428$

Indig D, Eyeson-Annan M, Copeland J, Conigrave KM (2007) The effects of alcohol consumption, psychological distress and smoking status on emergency department presentations in New South Wales, Australia. BMC Public Health 7:46

Jentsch JD, Woods JA, Groman SM, Seu E (2010) Behavioral characteristics and neural mechanisms mediating performance in a rodent version of the balloon analog risk task. Neuropsychopharmacology 35:1797-1806

Kalman D, Morissette SB, George TP (2005) Co-morbidity of smoking in patients with psychiatric and substance use disorders. Am J Addict 14:106-123

Kaufman J, Birmaher B, Brent D, Rao U, Flynn C, Moreci P, Williamson D, Ryan N (1997) Schedule for affective disorders and schizophrenia for school-age children-present and lifetime version (K-SADS-PL): initial reliability and validity data. J Am Acad Child Adolesc Psychiatry 36:980-988

Kirby KN, Petry NM, Bickel WK (1999) Heroin addicts have higher discount rates for delayed rewards than non-drug-using controls. J Exp Psychol Gen 128:78-87

Kypri K (2007) Methodological issues in alcohol screening and brief intervention research. Subst Abuse 28:31-42
Laird NM (1988) Missing data in longitudinal studies. Stat Med 7:305-315

Lejuez CW, Read JP, Kahler CW, Richards JB, Ramsey SE, Stuart GL, Strong DR, Brown RA (2002) Evaluation of a behavioral measure of risk taking: the Balloon Analogue Risk Task (BART). J Exp Psychol Appl 8:75-84

Lejuez CW, Aklin WM, Jones HA, Richards JB, Strong DR, Kahler CW, Read JP (2003) The Balloon Analogue Risk Task (BART) differentiates smokers and nonsmokers. Exp Clin Psychopharmacol 11:26-33

Lejuez CW, Aklin W, Bornovalova M, Moolchan ET (2005) Differences in risk-taking propensity across inner-city adolescent everand never-smokers. Nicotine Tob Res 7:71-79

Little RJA, Rubin DB (2002) Statistical analysis with missing data, 2nd edn. Wiley, New York

Myers MG, Kelly JF (2006) Cigarette smoking among adolescents with alcohol and other drug use problems. Alcohol Res Health 29:221-227

Patton JH, Stanford MS, Barratt ES (1995) Factor structure of the Barratt impulsiveness scale. J Clin Psychol 6:768-774

Pleskac TJ, Wallsten TS, Wang P, Lejuez CW (2008) Development of an automatic response mode to improve the clinical utility of sequential risk-taking tasks. Exp Clin Psychopharmacol 16:555564

Radloff LS (1977) The CES - a self-report depression scale for research in the general population. Appl Psychol Meas 1:385-401

Ray L, Ashenhurst J (2010) Risky decision making in individuals with alcohol use disorders: genetic and clinical moderators. 2010 Abstracts of the 33rd Annual Scientific Meeting of the Research Society on Alcoholism, 26-30 June, San Antonio, Texas, p 34

Ryb GE, Dischinger P, Kufera J, Soderstrom C (2007) Smoking is a marker of risky behaviors independent of substance abuse in injured drivers. Traffic Inj Prev 8:248-252

Schneider D, Morris J (1991) Risk-taking behaviors of college students. Environ Behav 23:575-591

Seamark CJ, Gray DJ (1998) Teenagers and risk-taking: pregnancy and smoking. Br J Gen Pract 48:985-986

Spielberger CD, Gorsuch RL, Lushene RD, Vagg PR, Jacobs GA (1983) State-trait anxiety inventory for adults: manual (form Y). Mind Garden, Redwood City

SRNT (2002) Biochemical verification of tobacco use and cessation. Nicotine Tob Res 4:149-159

Tull MT, Trotman A, Duplinsky MS, Reynolds EK, Daughters SB, Potenza MN, Lejuez CW (2009) The effect of posttraumatic stress disorder on risk-taking propensity among crack/cocaine users in residential substance abuse treatment. Depress Anxiety 26:1158-1164

Valois RF (1999) Relationship between number of sexual intercourse partners and selected health risk behaviors among public high school adolescents. J Adolesc Health 25:328-335

Wallsten TS, Pleskac TJ, Lejuez CW (2005) Modeling behavior in a clinically diagnostic sequential risk-taking task. Psychol Rev 112:862-880

Wechsler D (1999) Wechsler abbreviated scale of intelligence. The Psychological Corporation, San Antonio 